Relations industrielles

Industrial Relations

\title{
La psychologie et les Sciences humaines dans l'Entreprise, Louis-Marie Le Maitour, Les Editions d'Organisation, Paris, 1962.
}

\section{J.-P. Deschênes}

Volume 18, numéro 1, janvier 1963

URI : https://id.erudit.org/iderudit/1021471ar

DOI : https://doi.org/10.7202/1021471ar

Aller au sommaire du numéro

\section{Éditeur(s)}

Département des relations industrielles de l’Université Laval

\section{ISSN}

0034-379X (imprimé)

1703-8138 (numérique)

Découvrir la revue

Citer ce compte rendu

Deschênes, J.-P. (1963). Compte rendu de [La psychologie et les Sciences humaines dans l'Entreprise, Louis-Marie Le Maitour, Les Editions d'Organisation, Paris, 1962.] Relations industrielles / Industrial Relations, 18(1), 136-137. https://doi.org/10.7202/1021471ar

Tous droits réservés @ Département des relations industrielles de l’Université Laval, 1963
Ce document est protégé par la loi sur le droit d'auteur. L’utilisation des services d'Érudit (y compris la reproduction) est assujettie à sa politique d'utilisation que vous pouvez consulter en ligne.

https://apropos.erudit.org/fr/usagers/politique-dutilisation/ 
America's Forgotten Labor Organization. A Survey of the Role of the SingleFirm Independent Union in American Industry by Arthur B. Shostak. Industrial Relations Section. Princeton University, Princeton, New Jersey, 1962, 139 pp.

Aux Etats-Unis les travailleurs sont syndiqués dans différents genres de groupements.

On connaît assez bien la centrale AFL-CIO avec les grandes unions internationales et nationales qui lui sont affiliées. On connaît aussi certaines grandes unions indépendantes comme celles des Travailleurs des mines, des camionneurs et les Fraternités de Chemin de fer. En tout cas, il existe à leur sujet une multitude d'études analysant leur nature, leur rôle, leur structure et leur fonctionnement.

Cependant, aux Etats-Unis, le monde ouvrier organisé ne comprend pas exclusivement des unions affiliées. Il existe un certain nombre de groupements d'usine qui, ensemble, ont un effectif de plus de 400,000 travailleurs et jouent un certain rôle dans l'aménagement des relations patronales-ouvrières. Jusqu'ici, ce genre de groupements n'a pas donné lieu à des études systématiques de la part des observateurs des problèmes de relations du travail.

D'ailleurs, justement à cause de leur multiplicité, de leur morcellement, et parfois de la discrétion qui a entouré leur existence et leur action syndicale, elles ont moins attiré l'attention. Elles ont été oubliées. Souvent aussi elles ont été considérées simplement comme des syndicats jaunes, et pour cela, jugées négligeables.

L'étude menée par Arthur B. Shostak, America's Forgotten Labor Organization, sous les auspices de la section des relations industrielles de l'Université de Princeton vient combler cette lacune. Elle est brève mais particulièrement bien faite.

L'auteur définit ce qu'il entend par * Union indépendante dans une seule entreprise 》 (Single-Firm Independant Union), décrit ses caractères et la compare avec le groupement local d'une union affiliée. Il passe à tour de rôle les groupements de collets-bleus, de colletsblancs, des ingénieurs. Certaines de ces unions indépendantes se sont groupées en associations à un niveau supérieur à celui de l'usine; l'auteur analyse ce phénomène avant d'aborder les rapports entre les unions internationales et les unions indépendantes d'usine. Enfin dans sa conclusion il examine le futur de ces groupements.

De ses constatations il découle que ces Unions sont beaucoup plus indépendantes qu'on le croit ordinairement et qu'elles ont réussi à se maintenir et ren-

dre certains services malgré l'hostilité du syndicalisme organisé et de l'attitude des employeurs. Toutefois, elles donnent lieu à des paradoxes frappants : ce sont les unions les plus faibler qui ont 'e plus de sécurité, alors que les plus puissantes ont plus de chance de devenir la proie des grandes unions internationales. Cela est dû au fait que les relations patronales-ouvrières sont de plus en plus caractérisées par une consolidation des pouvoirs tant du côté des travailleurs que de celui des entreprises.

Les dirigeants de ces groupements, comme ceux des grandes unions et les employeurs tireront profit de la lecture de cet ouvrage impartial et bien documenté.

\section{Gerard Dion}

\section{La psychologie et les Sciences humaines} dans l'Entreprise, Louis-Marie Le Maitour, Les Editions d'Organisation, Paris, 1962.

Comme l'explique l'auteur dans son introduction, «un des intérêts de cet ouvrage nous semble résider dans le fait qu'en présentant un «panorama 》 des applications de la psychologie dans l'entreprise, il situe la place de chacune de ses applications par rapport aux autres et par rapport à l'ensemble \$.

Depuis Taylor, l'initiateur de ce qu'on appelle communément le \& génie industriel », on en est venu à considérer l'entreprise comme une organisation technique où l'homme est un rouage parmi d'autres, une machine, un peu spéciale toutefois, qui doit s'ajuster à d’autres machines. L'utilisation parfois à outrance des techniques de l'étude des mouvements et des temps en est une preuve. 
Peu à peu cependant on réalisa qu'un individu se distingue essentiellement d'un autre individu, et que les relations qui s'établissent entre les travailleurs ainsi qu'entre les travailleurs et les dirigeants sont d'une importance capitale dans la vie de l'entreprise.

Le volume de Le Maitour se divise en trois grandes sections. L'entreprise et ses exécutants, l'entreprise et ses dirigeants, l'entreprise et le public.

La première section analyse les difficultés qui doivent être aplanies afin d'intégrer plus facilement le travailleur dans l'entreprise. Ces difficultés d'ordre psychologique et physiologique, sont la fatigue, la monotonie et les changements technologiques. L'auteur propose ensuite une série de remèdes à la fatigue, à la monotonie, et, d'une façon plus générale, insiste sur l'importance de la sélection, des méthodes de la psychotechnique, de la formation professionnelle, de l'adaptation du travail à l'homme, de l'étude des attitudes collectives pour maintenir un bon moral dans l'organisation, etc.

La seconde section s'attache davantage à ceux qui, aux divers échelons, ont une fonction plus ou moins importante, plus ou moins large de direction, c'est-à- dire le personnel des «cadres》. Entre autres, les sujets qui y sont traités sont l'évolution de la philosophie du chef, les problèmes de la décision, les communications dans l'entreprise, la structure de l'entreprise (formelle et informelle), les problèmes de la maîtrise, la formation psychologique des cadres, ainsi que le jugement des travailleurs et du travail.

La dernière section analyse l'entreprise \& en contact avec d'autres entreprises, avec la presse, avec différents organismes régionaux et nationaux, bref avec la société tout entière dont elle ne constitue qu'une petite cellule $\gg$. En d'autres mots, c'est tout le problème des relations que doit établir l'entreprise qui sont étudiées.

Comme l'auteur le signale lui-même, le volume présente une vue générale de l'application de la psychologie dans l'entreprise. Une analyse plus poussée de cette application est du domaine du psychologue industriel, mais il n'en demeure pas moins que les problèmes soulevés dans ce travail doivent intéresser non seulement les dirigeants d'entreprises mais aussi tous ceux qui jouent un rôle quelconque dans une organisation.

\section{J.-P. Deschênes}

\section{PUBLICATIONS RÉCENTES RECENT PUBLICATIONS}

\section{Généralités}

«Labor Policy in a Changing World », by Richard A. Lester, Industrial Relations, Vol. 2, No. 1, October 1962, pp. 39-53.

«Para la empresa libre », por Martin Brugarola, S.J., Boletin De Divulgacion social, Num. 193, Septiembre 1962, pp.

« Para la empresa libre », por Matin Brugarola, S.J., Boletin De Divulgacion social, Num. 193, Septiembre 1962, pp. 466-468.
« Evolution démographique et polarisation de la région de Montréal », par Claude Germain, L'Actualité Economique, no 2, juillet-septembre 1962, pp. 245-277.

\& The Functioning of the Market for Economists », by Gerald G. Somers, Reprint Series No. 31, The Industrial Relations Research Center, The University of Wisconsin.

«Bibliography of British Labor and Radical Journal 1880-1914 », by Jacqueline Brophy, Reprint Series No. 43, 